\title{
Instability of the interface between joint conducting materials under electrical current
}

\author{
R.V. Goldstein ${ }^{1}$, T.M. Makhviladze 2 , M.E. Sarychev ${ }^{\dagger, 2}$ \\ ${ }^{\dagger}$ sarych@yandex.ru \\ ${ }^{1}$ Institute for Problems in Mechanics, RAS, Vernadsky Prst. 101/1, 119526, Moscow \\ ${ }^{2}$ Institute of Physics and Technology, RAS, Nakhimovskii Prst. 36/1, 117218, Moscow
}

\begin{abstract}
The problem of improving the reliability of contact connections of metals in modern micro- and nanoelectronic devices is associated with the influence of electromigration processes on the stability of the shape of conductive materials interface under the current load. The interface between the two materials can become unstable under the influence of ion electromigration. In the present work, model describing instability of a flat interface between conducting materials under electromigration processes along the interface and in the materials bulks is developed. The dependences of the instability conditions on a value and direction of the electrical current in the number of cases, when the bulk electromigration plays the main role, are obtained and analyzed. Analysis of the relations in the case of identical materials shows the principal possibility of interface instability due to the bulk electromigration.
\end{abstract}

Keywords: vacancies, electromigration, diffusion.

\section{Неустойчивость границы соединенных проводящих материалов под действием электрического тока}

\author{
Гольдштейн Р.В. ${ }^{1}$, Махвиладзе T.M. ${ }^{2}$, Сарычев M.Е. ${ }^{\dagger, 2}$ \\ ${ }^{\dagger}$ sarych@yandex.ru \\ ${ }^{1}$ Институт проблем механики им. А.Ю. Ишлинского РАН, пр. Вернадского, 101/1, 119526, Москва \\ ${ }^{2}$ Физико-технологический институт РАН, Нахимовский пр., 36/1, 117218, Москва
}

Проблема повышения надежности работы контактных соединений металлов в современных микро- и наноэлектронных устройствах связана с задачей влияния электромиграционных процессов на устойчивость формы проводящих материалов под токовой нагрузкой. Граница между двумя материалами может оказаться неустойчивой под действием ионной электромиграции. В данной работе развита модель, описывающая потерю устойчивости плоской границы между проводящими материалами под действием процессов электромиграции в границе и в объеме материалов. Получены и исследованы зависимости условий возникновения неустойчивости от величины и направления электрического тока в ряде случаев, когда основную роль в ее развитии играет объемная электромиграция. Анализ полученных соотношений для случая одинаковых материалов показывает принципиальную возможность возникновения неустойчивости границы за счет объемной электромиграции.

Ключевые слова: вакансии, электромиграция, диффузия.

\section{1. Введение}

В связи с проблемой повышения надежности работы межсоединений в современных микро- и наноэлектронных устройствах значительное внимание уделяется теоретическим исследованиям влияния электромиграционных процессов на устойчивость формы проводящих материалов под токовой нагрузкой [1-3]. В частности, в [1] рассматривалась возможность возникновения не- устойчивости границы (интерфейса) между двумя материалами под действием ионной электромиграции в ней. В [2,3] показано, что объемный электромиграционный транспорт вакансий в некоторых условиях может играть определяющую роль в развитии неустойчивости формы свободной поверхности проводящего слоя на подложке.

В настоящей работе предложена модель, описывающая развитие неустойчивости формы интерфейса двух проводящих материалов при учете одновременного дей- 
ствия как ионной электромиграции в нем, так объемной вакансионной электромиграции в материалах. Получены и исследованы уравнения, описывающие кинетику пространственно-периодических возмущений интерфейса. Найдены и аналитически исследованы условия, при которых объемная электромиграция может превалировать над электромиграцией в интерфейсе, приводя к неустойчивости его плоской формы. В ряде случаев даны оценки длин волн возмущений и плотностей протекающего тока, при которых может реализоваться такая ситуация.

\section{2. Основные соотношения модели}

Рассмотрим бесконечно протяженный плоский интерфейс (плоскость $(x, z))$ двух проводящих монокристаллических материалов 1 и 2. Будем считать оба материала бесконечно протяженными в плоскости $(x, z)$ и полубесконечными вдоль оси $y$, перпендикулярной к интерфейсу (ось $y$ направлена вверх, от материала 1 к 2, а $y=0$ отвечает плоскости интерфейса). Считаем также, что все величины, относящиеся к соединенным материалам, не зависят от $z$. Пусть перпендикулярно интерфейсу в направлении от 1 к 2 действует электрическое поле, вызывающее электрический ток с плотностью .

В данных условиях рассмотрим кинетику возмущения плоского интерфейса, которое имеет вид

$$
h(x, t)=A(t) \sin \omega x
$$

где $h$ - изменение профиля интерфейса вдоль оси $y$, $A-$ амплитуда, зависящая от времени $t, \omega=2 \pi / \lambda>0$ и $\lambda$ - длина волны. Амплитуда $A(t)$ предполагается малой по сравнению с $\lambda$, поэтому далее ограничимся при анализе линейным по $A(t)$ приближением.

Возмущение (1) приводит к возникновению упругих деформаций и механических напряжений в материалах 1 и 2. Эти деформации будут влиять на потоки ионов вдоль интерфейса [1] и вакансий из объемов материалов [4], которые, в свою очередь, оказывают на них обратное воздействие. В результате в приближении состояния плоской деформации [1,2] получим, что этот процесс описывается следующей системой уравнений:

$$
\begin{gathered}
\frac{\partial u}{\partial t}=-\frac{\partial}{\partial x}\left(J_{1}+J_{2}\right)-\left(\Omega_{1} J_{1}^{(\mathrm{V})}+\Omega_{2} J_{2}^{(\mathrm{V})}\right) \\
\frac{\partial h}{\partial t}=-\frac{\partial}{\partial x}\left[\xi J_{1}+(1-\xi) J_{2}\right]-\left[\Omega_{1} J_{1}^{(\mathrm{V})} \xi-(1-\xi) \Omega_{2} J_{2}^{(\mathrm{V})}\right]
\end{gathered}
$$

где

$$
u=u_{1}+u_{2}=U(t) \sin \omega x
$$

$u_{1,2}$ - упругое смещение границ интерфейса со стороны материала 1 и 2, соответственно, $\xi=G_{1} /\left(G_{1}+G_{2}\right)$, $G_{1,2}=E_{1,2} /\left(1-v_{1,2}^{2}\right), E_{1,2}$ и $v_{1,2}-$ модули Юнга и коэффициенты Пуассона материалов, $J_{1,2}$ и $J_{1,2}^{(\mathrm{V})}-$ потоки объема за счет ионного массопереноса вдоль интерфейса и переноса вакансий из объемов материалов к интерфейсу, возникающие вследствие возмущения (1); $\Omega_{1,2}-$ удельные вакансионные объемы в 1 и 2.
В уравнениях (2) потоки $J_{1,2}$ имеют тот же вид, что и в [1], а потоки $J_{1,2}^{(\mathrm{V})}$ включают диффузионный, электромиграционный и механический вклады

$$
\begin{aligned}
J_{p}^{(\mathrm{V})}=-D_{p} & {\left[\frac{\partial\left(C_{p} / \Omega_{p}\right)}{\partial y}+\frac{\left(C_{p} / \Omega_{p}\right) Z_{p}^{(\mathrm{V})}}{k T} \frac{\partial \varphi_{p}}{\partial y}+\right.} \\
& \left.+\frac{\left(C_{p} / \Omega_{p}\right)}{k T} \Omega_{p} f_{p} \frac{\partial \sigma_{S, p}}{\partial y}\right]\left.\right|_{y=0}, \quad p=1,2
\end{aligned}
$$

где $D_{p}$ и $C_{p}-$ коэффициенты диффузии и безразмерные (относительные) концентрации вакансий, $Z_{p}^{(\mathrm{V})}>0-$ их эффективный заряд $[2,4], \varphi_{p}=\varphi_{p}(x, y)-$ распределение электрического потенциала в соединенных материалах [1], $\sigma_{S, p}$ - шаровая часть тензора механических напряжений в материалах, обусловленное возмущением (1), $f_{p}$ - соответствующий коэффициент релаксации вакансий [4], T- абсолютная темперапура, $k$ - постоянная Больцмана.

Используя для распределений $\varphi_{p}=\varphi_{p}(x, y)$ и распределений компонент тензора механических напряжений результаты [1], с учетом малости возмущения (1) и соответственно величин $\sigma_{s, p}$ и $u$, можно получить, что распределения концентраций вакансий в каждом из материалов должны находиться из уравнений:

$$
\frac{\partial C_{p}}{\partial t}=D_{p}\left(\frac{\partial^{2} C_{p}}{\partial x^{2}}+\frac{\partial^{2} C_{p}}{\partial y^{2}}\right)-\frac{D_{p} Z_{p}^{(\mathrm{V})} \rho_{p} j}{k T} \frac{\partial C_{p}}{\partial y}
$$

где $\rho_{p}-$ удельное сопротивление материала. Решая это уравнение в стационарном режиме $\left(\partial C_{p} / \partial t=0\right)$ методом разделения переменных (см. также $[2,3])$, получим

$$
\begin{aligned}
C_{p}(x, y)=C_{p}^{(e)}- & \frac{C_{p}^{(e)} \Omega_{p}}{k T}\left[ \pm \gamma \omega^{2} A-2 / 3\left(1+v_{p}\right) G \omega U+\right. \\
& \left.+\left(Z_{p}^{(\mathrm{V})} j \rho / \Omega_{p}\right) A\right] \sin (\omega x) \exp \left(q_{ \pm} y\right)
\end{aligned}
$$

где $C_{p}^{(e)}-$ равновесные концентрации вакансий в материалах при плоской границе, $q_{ \pm}=\left(\alpha_{p} \pm \sqrt{\alpha_{p}^{2}+4 \omega^{2}}\right) / 2$, $\alpha_{p}=Z_{p}^{(V)} E / k T$ и знак «土» в (5) отвечает $p=1,2$ соответственно ( $\left.\mathrm{q}_{+}>0, \mathrm{q}_{-}<0\right), \rho=2 \rho_{1} \rho_{2} /\left(\rho_{1}+\rho_{2}\right), \rho_{1,2}-$ удельные сопротивления материалов, $\gamma-$ коэффициент поверхностного натяжения интерфейса, а $G=2 G_{1} G_{2} /\left(G_{1}+G_{2}\right)$ и считается, что $C_{2,1}(x, y \rightarrow \pm \infty)=C_{2,1}^{(e)}$. При нахождении решения (5) интерфейс рассматривается как поверхность, адсорбирующая точечные дефекты из объемов материалов $[5,6]$, в силу чего для каждого из материалов можно воспользоваться граничным условием в виде $C_{p}(x, y=0)=C_{p}^{(e)} \exp \left(\Delta \mu_{p} / k T\right)$ (термодинамическое соотношение Гиббса-Томсона [7]), где $\Delta \mu_{p}$ - изменение химического потенциала вакансий $р$-го материала в интерфейсе за счет возмущения (1).

Подставляя теперь ссоотношения (1), (3), (4) и выражения для потоков $J_{1,2}$ из [1] в уравнения (2), в линейном по амплитудам $A(t)$ и $U(t)$ приближении получим для них систему уравнений:

$$
\begin{aligned}
& \mathrm{d} A / \mathrm{d} t=b_{11} A+b_{12} U \\
& \mathrm{~d} U / \mathrm{d} t=b_{21} A+b_{22} U
\end{aligned}
$$

где коэффициенты $b_{i j}(i, j=1,2)$, могут быть полностью 
выписаны в общем случае через параметры задачи, но представляют собой слишком громоздкие для формата данной статьи выражения (они приведены в [8]).

Стандартное решение системы (6) дает

$$
\begin{aligned}
& A(t)=A(0)\left[g \exp \left(\zeta_{+} t\right)+(1-g) \exp \left(\zeta_{-} t\right)\right] \\
& U(t)=U(0)\left[s \exp \left(\zeta_{+} t\right)+(1-s) \exp \left(\zeta_{-} t\right)\right]
\end{aligned}
$$

где $\{g, s\}-$ собственный вектор матрацы $b_{i j}$, а $\zeta_{ \pm}$ее собственные числа:

$$
\zeta_{ \pm}=\frac{b_{11}+b_{22}}{2} \pm \sqrt{\left(\frac{b_{11}+b_{22}}{2}\right)^{2}-\left(b_{11} b_{22}-b_{12} b_{21}\right)}
$$

Таким образом, из выражений (7), (8) следует, что форма интерфейса будет неустойчивой относительно возмущений (1), если, по крайней мере, одна из величин $\zeta_{+}=\zeta_{+}(\omega)$ окажется положительной. Соответственно, для устойчивости плоского интерфейса необходимо, чтобы оба собственных числа $\zeta_{ \pm}$были отрицательны.

\section{3. Анализ условий устойчивости; некоторые оценки}

Из (8), в частности, видно, что положительность $\zeta_{ \pm}$(неустойчивость интерфейса), во всяком случае, для корня $\zeta_{+}$в (8), имеет место при выполнении условия

$$
b_{11} b_{22}-b_{12} b_{21}<0
$$

Соотношение (9) является простейшим достаточным условием расходимости со временем выражений (7). В принципе, исходя из выражения (8), можно записать и общие условия на величины $b_{i j}$, но здесь остановимся только на выполнимости (9). Наибольший интерес представляет выяснить, может ли это происходить за счет вакансионной электромиграции.

Ввиду громоздкости выражений для коэффициентов $b_{i j}$ общий анализ условий устойчивости интерфейса здесь не представляется возможным. Поэтому рассмотрим значительно более простой, но достаточно показательный частный случай, когда интерфейс образован одинаковыми материалами. Тогда соотношения для коэффициентов $b_{i j}$, полученные в [8], записываются следующими компактными выражениями, допускающими аналитическое исследование:

$$
\begin{aligned}
& b_{11}=-\omega^{4} \gamma L+3 \omega^{2} \gamma V \alpha / 2-V \bar{Z}_{V} j \rho \sqrt{\alpha^{2} / 4+\omega^{2}}, \\
& b_{12}=-G(1+v) V \omega\left(f \omega-4 \sqrt{\alpha^{2} / 4+\omega^{2}}\right) / 6, \\
& b_{21}=\omega^{2}\left[2 \bar{Z}^{*} j \rho L-2 \gamma V \sqrt{\alpha^{2} / 4+\omega^{2}}\right]-3 V \bar{Z}_{V} j \rho \alpha, \\
& b_{22}=-2 \omega^{3} G L+2 \omega(1+v) G V \alpha,
\end{aligned}
$$

где $L=\delta D_{I} \Omega / k T, V=D \bar{C}_{e} \Omega / k T, \delta-$ ширина интерфейса, $D_{I}$ - коэффициент диффузии ионов материала в интерфейсе, $D=D_{1}=D_{2}, \bar{C}_{e}=C_{1}^{(e)}=C_{2}^{(e)}, \Omega=\Omega_{1}=\Omega_{2}, \alpha=\alpha_{1}=\alpha_{2}$, $\bar{Z}^{*}=Z^{*} / \Omega, Z^{*}=Z_{1}^{*}=Z_{2}^{*}<0-$ эффективный заряд ионов материала в интерфейсе [4], $\bar{Z}_{V}=Z_{V} / \Omega, Z_{V}=Z_{1}^{(\mathrm{v})}=Z_{2}^{(\mathrm{v})}$, $v=v_{1}=v_{2}, f=f_{1}=f_{2}<1, \rho=\rho_{1}=\rho_{2}$. Отметим, что этот случай может представить и самостоятельный интерес, например, при исследовании влияния электромиграции на устойчивость межзеренных границ в поликристаллических микропроводниках.

В выражениях (10) коэффициент $b_{12} \sim V$, т.е. он отличен от нуля только за счет учета объемной электромиграции. Без этого $b_{12}=0$ и, как следует из $(8), \zeta_{+}=b_{11}<0$ и $\zeta_{-}=b_{22}<0$, т.е. интерфейс заведомо устойчив. Кроме того, из (10) следует, что $b_{12}>0$ при любых значениях $\omega$ и $j$.

Остальные коэффициенты наиболее полно могут быть проанализированы в двух предельных случаях $|\alpha| \gg \omega$ и $|\alpha| \ll \omega$, когда выражения (10) представляют собой полиномы по степеням $\omega$. В настоящей работе ограничимся рассмотрением первого из них, как более простого, имея в виду прежде всего оценку знаков выражений (10) в различных диапазонах плотностей токов и длин волн возмущения (1).

При $|\alpha| \gg \omega$ из (10) сразу имеем, что $b_{22}$ может менять знак при $\omega=\omega_{1}(j)$, где

$$
\omega_{1}=\sqrt{(1+v) \beta \alpha},
$$

$b_{21}-$ при $\omega^{2}=\omega_{2}^{2}>0$, где

$$
\omega_{2}^{2}=\frac{3 \beta \bar{Z}_{V} j \rho \alpha}{2 \bar{Z}^{*} j \rho-\gamma \beta|\alpha|},
$$

и $b_{11}-$ при $\omega=\omega_{3}$, где $\omega_{3}^{2}-$ положительный корень уравнения $b_{11}=0$ :

$$
\omega_{3}^{2}=\frac{3}{4} \beta \pm \sqrt{\frac{9}{16} \beta^{2}-\beta\left(\bar{Z}_{V} j \rho\right)|\alpha| / 2 \gamma},
$$

где $\beta=V / L$.

Оценим выражения (11), например, для межзеренных границ в поликристаллах алюминия (Al). В этом случае $\delta \approx(2 \div 5) \times 10^{-10} \mathrm{M}, \gamma \approx 1$ Дж/ $\mathrm{M}^{2}, \Omega \approx 2 \times 10^{-29} \mathrm{M}^{3}$ [9], $Z_{V}=\left|Z^{*}\right| \sim 10 e, e=1.6 \times 10^{-19} \mathrm{Kл}-$ заряд электрона $[2,9]$, $v \approx 0.3, f \approx 0.4, \rho \approx 3 \times 10^{-8}$ Ом м [9] и, согласно данным [10, 11] при $T=100^{\circ} \mathrm{C}\left(k T=0.032\right.$ эВ) имеем $D_{I} \approx 6 \times 10^{-15} \mathrm{M}^{2} / \mathrm{c}$, $D \approx 1.5 \times 10^{-23} \mathrm{M}^{2} / \mathrm{c}$, т.е. $\beta=V / L \approx 5 \times 10^{-9} 1 / \mathrm{M}$. Аналогично при $T=350^{\circ} \mathrm{C}(k T=0.045$ эВ $)-D_{I} \approx 5 \times 10^{-11} \mathrm{M}^{2} / \mathrm{c}$, $D \approx 10^{-15} \mathrm{M}^{2} /$ c, т.е. $\beta \approx 10^{-2} 1 / \mathrm{M}$. Отметим, что выбранные для оценок температуры $T=100^{\circ} \mathrm{C}$ и $350^{\circ} \mathrm{C}$ интересны, в частности, тем, что именно в этом температурном интервале проводятся ускоренные эксперименты по исследованию надежности микроэлектронной металлизации $[4,10]$.

Используя эти данные, получим оценку параметра $\alpha \approx 10^{-5} j(1 / \mathrm{M})\left([j]=\mathrm{A} / \mathrm{M}^{2}\right)$. Тогда исходное условие $|\alpha| \gg \omega$ дает связь отвечающих ему диапазонов длин волн возмущения и плотностей токов

$$
\lambda \gg \lambda_{c} \equiv 2 \pi /|\alpha|=6 \times 10^{5} /|j| \mathrm{M} .
$$

Отсюда, например, для плотностей тока $j=10^{10} \mathrm{~A} / \mathrm{m}^{2}$, имеем $\lambda \gg \lambda_{c}=10^{2}$ мкм.

Соответственно, из (11a) при $T=350^{\circ} \mathrm{C}$ имеем $\lambda_{1} \approx 10^{4} / V_{j}$ м и для той же плотности тока $\lambda_{1} \approx 0.1 \mathrm{м}$, много больше $\lambda_{c}$; при $\lambda<\lambda_{1}$ коэффициент $b_{22}<0$.

Учитывая равенство $Z_{V}=\left|Z^{*}\right|$, выражение (116) можно привести к виду

$$
\omega_{2}^{2}=-\frac{3 \beta(k T / \Omega) \alpha}{2(k T / \Omega)+\gamma \beta \operatorname{sgn}(\alpha)},
$$


откуда, используя те же значения параметров, что выше, при $T=350^{\circ} \mathrm{C}$ получим оценку $\omega_{2}^{2} \approx-\beta \alpha \sim-10^{-7} j$. Таким образом, при $j<0$ (ток направлен из материала 2 в 1) корень $\omega_{2}$ существует и $\lambda_{2} \approx 3 \times 10^{3} / \sqrt{ }|j|$, что при $|j|=10^{10} \mathrm{~A} / \mathrm{M}^{2}$ дает $\lambda_{2} \approx 3 \times 10^{-2} \mathrm{M} \gg \lambda_{c}$. Соответственно при $\lambda<\lambda_{2}$ коэффициент $b_{21}>0$.

Оценивая теперь $\omega_{3}$ и знак коэффициента $b_{11}$, будем сразу считать, что $j<0$. Тогда из (11в) для положительного корня имеем выражение

$$
\omega_{3}^{2}=\frac{3}{4} \beta+\sqrt{\frac{9}{16} \beta^{2}+\beta(k T / \Omega)|\alpha|^{2} / 2 \gamma},
$$

что при $T=350^{\circ} \mathrm{C}$ и $|j|=10^{10} \mathrm{~A} / \mathrm{M}^{2}$ дает $\omega_{3} \approx 10^{4} 1 / \mathrm{M}$, т.е. $\lambda_{3} \approx 600$ мкм $>\lambda_{c}=10^{2}$ мкм. При $\lambda>\lambda_{3}$, т.е. $\omega<\omega_{3}$, из $(10)$ имеем $b_{11}>0$.

Таким образом, сделанные оценки показывают, что при плотности тока $|j|=10^{10} \mathrm{~A} / \mathrm{M}^{2}$, направленного от материала 2 к 1, для возмущений вида (1) с длинами волн, например, в интервале 600 мкм $\leq \lambda \leq 10^{3}$ мкм имеет место $b_{12}>0, b_{21}>0, b_{22}<0$ и $b_{11}>0$, т.е. заведомо выполняется условие (9), Следовательно, корень $\zeta_{+}$в (7) положителен и такие возмущения будут нарастать со временем, т.е. плоская форма интерфейса относительно них будет неустойчивой.

\section{4. Заключение}

Развита модель, описывающая влияние электромиграции вакансий, протекающей в объеме проводящих соединенных материалов под действием электрического тока, на устойчивость формы плоской границы (интерфейса) между ними. Сформулирована и решена система уравнений, описывающая взаимосвязь изменений профиля интерфейса и механических напряжений, которые возникают за счет потоков ионов и вакансий, инициированных малым пространственно-периодическим возмущением интерфейса. Найдены критерии нарастания амплитуды таких возмущений со временем, т.е. условия неустойчивости формы интерфейса.

Для случая одинаковых материалов аналитически выделены области значений длин волн возмущений и плотностей тока, в которых именно объемная электромиграция вакансий приводит к росту со временем амплитуды возмущения, т.е. изначально плоский интерфейс становится неустойчивым. Для таких областей по длинам волн получены условия их возникновения и зависимости граничных значений от величины и направления электрического тока. Даны оценки одного из возможных диапазонов длин волн возмущения, при которых возникает неустойчивость интерфейса. Отметим, что эти асимптотические оценки показывают принципиальную возможность возникновения неустойчиво- сти границы за счет объемной электромиграции при достаточно разумных (для экспериментов) значениях основных параметров системы $T \sim 10^{2}\left({ }^{\circ} \mathrm{C}\right),|j| \sim 10^{10} \mathrm{~A} / \mathrm{M}^{2}$ и $\lambda \sim\left(10^{2} \div 10^{3}\right)$ мкм.

В случае разных материалов (он будет подробнее рассмотрен в следующих работах) аналитический анализ значительно более громоздок. Однако полученные в настоящей работе результаты, дают определенные основания утверждать, что и при этом возможны условия, в которых объемная электромиграция будет, по крайней мере, значительно влиять на кинетику развития возмущений профиля интерфейса.

Результаты работы могут оказаться полезными, например, для совершенствования технологических процессов производства микро- и наноэлектронных структур и устройств, а также для обеспечения их более надежного функционирования.

\section{Литература/References}

1. L. Klinger, L. Levin, O. Srolovitz. J. Appl, Phys, 79. 68346839 (1996).

2. R.V. Goldstein, T.M. Makhviladze, M.E. Sarychev. Letters on materials. 42(3). 171 (2014) (in Russian) [P.B. Гольдштейн, Т.М. Махвиладзе, М.Е. Сарычев. Письма о материалах. 42(3). 171 (2014)].

3. R.V. Goldstein, T.M. Makhviladze, M.E. Sarychev Surface. 1. 1 (2015) [Р.В. Гольдштейн, Т.М. Махвиладзе, М.Е. Сарычев. Поверхность. N 1.1 (2015)].

4. K.A. Valiev, R.V. Goldstein. Yu.V. Zhitnikov et al. Microelektronika. 38. 364 (2009) (in Russian) [К.A. Валиев, Р.В. Гольдштейн, Ю.В. Житников и др. Микроэлектроника. 38. 364 (2009)].

5. B.S. Bokstein, Ch.V. Kopetskii, P.S. Shvindlerman. Thermodynamics and kinetics of grain boundaries in metals. Moscow. Mtallurgy (1986). 222 p. (in Russian).

6. R.V. Goldstein, T.M. Makhviladze, M.E. Sarychev. Surface. N 8.5 (2011) [Р.В. Гольдштейн, Т.М. Махвиладзе, М.Е. Сарычев. Поверхность. N 8.5 (2011)].

7. Yu.M. Petrov. Clusters and small particles. Moscow. Nauka (1986). 367 p. (in Russian) [Ю.М. Петров. Кластеры и малые частицы. Москва. Наука. 1986. 367 с.].

8. R.V. Goldstein, T.M. Makhviladze, M.E. Sarychev. Preprint IPM RAS. № 1106 (2015). 11 p. (in Russian)

9. Handbook of Physical Quantities, ed. by Grigoriev I.S. and Meilikhov E.Z., Moscow. Energoatomizdat (1991) (in Russian).

10. K.N. Tu. J. Appl. Phys. 94. 5452-5473 (2003).

11. V.M. Chebatin. Physical chemistry of solids. Moscow. Khimia. (1982). 320 p. (in Russian) [В.М. Чебатин. Физическая химия твердых тел. Москва. Химия. 1982.320 c.]. 